\title{
Liver Injury and Khat Leaves: A Common Toxic Effect
}

\author{
Mona Abd Elmonem Hegazy, Nehad Mohamed Tawfik, Hoda Abd-Elstar Elrawi
}

\begin{abstract}
Background: The evidence of hepatotoxic effects of khat is often based on limited numbers of case reports and animal studies.
\end{abstract}

Aim: To evaluate the prevalence and severity of effects of khat on the liver.

Materials and methods: A total of 214 khat users, Yemeni people, included in the study. They were divided into two groups according to the presence or absence of hepatic insult either detected by elevated liver enzymes and/or abnormal hepatic finding on abdominal ultrasonography. Full medical history, clinical examination and calculation of body mass index (BMI) were done. Fasting blood sugar, total lipid profile, liver enzymes were estimated in all patients. Serum albumin, bilirubin and prothrombin time were assessed in patients showing positive hepatic damages (either by elevated liver enzymes and liver ultrasound). Abdominal ultrasound scan was done for all patients.

Results: One hundred and forty-four (group I) khat users showed a positive sign of hepatic injury either by elevated liver enzymes in 127 patients and abnormal hepatic scan finding in 89 patients. Four patients $(3 \%)$ discovered to have liver cirrhosis. Patients showed hepatotoxic effect of khat were significantly older in age than participants without hepatic affection. Both subgroups with variable BMI showed elevated ALT with no significant difference. Liver ultrasound showed positive hepatic insult in $40 \%$ of khat chewing patients with normal BMI, meaning that obesity is not the responsible factor for fatty liver in the current study.

Conclusion: Hepatotoxic effect of khat is common. Confounding issues other than khat were eliminated in the present study as causative factors, such as alcohol consumption, coexisting disease. Also, the effect of obesity was evaluated.

Abbreviations: BMI: Body mass index; LDL-C: Low density lipoprotein cholesterol; HDL-c: High density lipoprotein cholesterol; TG: Triglycerides; ALT: Alanine aminotransferase; AST: Aspartate aminotransferase.

Keywords: Khat, Hepatic injury, Liver ultrasound, Yemeni people.

How to cite this article: Abd Elmonem Hegazy M, Tawfik NM, Abd-Elstar Elrawi H. Liver Injury and khat Leaves: A Common Toxic Effect. Euroasian J Hepato-Gastroenterol 2012;2(2): 70-75.

\section{Source of support: Nil}

Conflict of interest: None

\section{INTRODUCTION}

Chewing the leaves of khat or Qat (Catha edulis Forsk) is a social habit in Yemen and East Africa countries. This habit has now spreading to ethnic communities in the rest of the world. At present, easy transportation of khat and easing of importation restrictions has helped this habit spread to countries, such as the USA and Western Europe, where
Yemeni, Somali and other East Africa communities are living. ${ }^{1}$ Figures for the numbers of individuals who chew khat in the general population of individual countries are mainly anecdotal. However, surveys have been performed to determine the exact incidence of khat chewing among specific cohorts. Fifteen years ago, Kalix P mentioned that there are six million daily khat users. ${ }^{2}$ Khat chewing among male Aden University medical students in Yemen increased from 35 to $90 \%$ over the 5 years of training. ${ }^{3}$ In college and high school students of Jazan region in Saudi Arabia aged between 15 and 25 years, 37.7\% of males and 3.8\% of females chewed khat. ${ }^{4}$ Also $15.9 \%$ of a sample of 4,001 men in Addis Ababa, Ethiopia regularly chewed khat. ${ }^{5}$ The prevalence of khat chewing in Western countries appears to be restricted to the immigrant communities from these countries where there is still a high prevalence among the immigrant groups. ${ }^{1}$ In the UK, 75 male Yemeni adults reported chewing up to three bundles of khat/week of them $39 \%$ were assessed as dependent. ${ }^{6}$ But, there is some evidence that khat use has spreaded to the host population, e.g. a pill containing extract of khat leaves known as 'Hagigat' has been sold to Israeli drug users. ${ }^{7}$ It is also spreading to nonethnic users in the UK. ${ }^{8}$ Khat is illegal in the USA, Canada and many European countries, including Denmark, Finland, Ireland, France, Germany, Switzerland, Norway and Sweden.

The pleasure derived from khat chewing is attributed to the euphoric actions of its content of 5-cathinone, a sympathomimetic amine, with properties described as similar to those of amphetamine. ${ }^{9}$ And, traditionally khat chewing has been viewed as an aid to relieving fatigue and has some place in self-medication of depression. ${ }^{10}$ However, the WHO concludes that it has no therapeutic potential. ${ }^{11}$ While the psychological effects of chronic khat use have been the subject of much debate on its influence of social structure, there is now mounting causes for concern over the health effects on a wide range of peripheral organs. Links have been proposed between khat chewing and the incidence of myocardial infarction, dilated cardiomyopathy, vascular diseases, such as hypertension, cerebrovascular ischemia and thromboembolism, diabetes, sexual dysfunction, duodenal ulcer and hepatitis. ${ }^{12}$

Hepatic cirrhosis of unknown etiology has been noted in khat users; poor diet and the potential hepatotoxic effects of khat Tannians may be contributing factors. ${ }^{13}$ The evidence, however, is often based on limited numbers of case reports $^{13-15}$ and animal studies. ${ }^{16,17}$ 
The aim of the current study is to focus on the prevalence and severity of hepatic affection in khat users' Yemeni people.

\section{MATERIALS AND METHODS}

\section{Study Design}

A retrospective descriptive observational cross-sectional study.

\section{Subjects}

The present study was conducted on Yemeni patients attending outpatient clinic, Internal Medicine Department, Kasar Al-Aini Hospital, Cairo University, Egypt. Over a 5 years period (September 2005-2010), either complaining of symptoms suggestive of irritable bowel syndrome, common cold or just for check-up, whom gave history of khat chewing were included in the study.

The selection of participants in the current study was based on the following inclusion criteria: All participants were male and female chewing khat, at any age. Patients with history of drinking alcohol or hepatitis $\mathrm{B}$ virus and hepatitis $\mathrm{C}$ virus infection were excluded from the study.

A total of 214 Yemeni patients were included in the current study. They were divided into two main groups: Group I included 144 patients with either elevated liver enzymes and/or abnormal hepatic finding in liver ultrasound examination. Group II included 70 patients with normal liver enzymes and normal liver scan. Further subdivision of group I according to body mass index (BMI) into group Ia; patients with normal BMI (18.5-25 kg/m²) and group Ib; patients with variable degree of overweight and obesity (25-40 kg/m²). Written consent was taken from the patients after explanation of the aim of the study.

\section{Methods}

All the 214 participants were interviewed for their medical history, undergone complete physical examination, the weight and height of each participant were measured and the BMI was calculated $\left(\mathrm{kg} / \mathrm{m}^{2}\right)$.

Blood samples were obtained from each participant after a fasting period of 10 to 12 hours for measuring: Fasting blood glucose, total cholesterol, low density lipoprotein cholesterol (LDL-c), high density lipoprotein cholesterol (HDL-c), triglycerides (TGs), liver enzymes, alanine aminotransferase (ALT), and aspartate aminotransferase (AST), complete blood picture, and creatinine.

Patients in group I however, showed either elevated liver enzyme or abnormal liver finding in ultrasound scan during checkup further tested for serum albumin, bilirubin and prothrombin time.
Abdominal ultrasound scan was performed to all participants. All ultrasound scans were performed using a high resolution B-mode scanner (SDD-5500, Aloka, Tokyo, Japan) $3.5 \mathrm{MHz}$ transducer.

The 144 patients (group I) discovered to have elevated liver enzymes and/or abnormal liver finding in ultrasound scan were tested for HCV and HBV antibodies.

\section{Statistical Methods used}

Data were statistically described in terms of range, mean \pm standard deviation ( \pm SD), and frequencies (number of cases) and relative frequencies (percentages) when using MannWhitney U-test for independent samples. A probability value (p-value) less than 0.05 was considered statistically significant. All statistical calculations were done using computer programs Microsoft Excel version 7 (Microsoft Corporation, NY, USA) and SPSS (Statistical Package for the Social Science; SPSS Inc., Chicago, IL, USA) version 15 for Microsoft Windows.

\section{RESULTS}

A total of 214 patients were included in the study. Group I included 144 patients, 123 males (85\%) and 21 (15\%) females, mean age of $39.9 \pm 10.39$ years. One hundred and twenty-seven patients from group I (88\%) had elevated liver enzymes, and 89 patients (62\%) showed abnormal hepatic finding during routine abdominal ultrasound scan. Group II included 70 patients, 55 males (79\%) and 21 females (15\%), mean age of $34.5 \pm 13$ years, with normal range of liver enzymes and normal liver ultrasound scan. All the clinical and laboratory data of both groups are showed in Table 1.

Group I patients with positive liver insult further subdivided according to BMI into group Ia patients, with normal BMI; included 55 patients, 51 males (93\%) and four females (7\%), and group Ib patients with variable degree of overweight and obesity; included 89 patients, 72 males (81\%) and 17 females (19\%). None of our patients had renal impairment, and three patients had normocytic norm chromic anemia, one patient had low platelets count and discovered to have liver cirrhosis.

The clinical and laboratory data of groups Ia, and Ib are showed in Table 2.

Four patients (3\%) discovered to have liver cirrhosis; their mean age was $54.75 \pm 7.12$ years, two patients had splenomegaly and one patient had ascites. Results of liver ultrasound scan in groups I, Ia and Ib patients are showed in Table 3. Patient's results of bright liver differ from fatty hepatomegaly in that; bright liver mean fat deposition but the size of the liver is not enlarged as in fatty hepatomegaly. 


\begin{tabular}{lccc}
\multicolumn{4}{c}{ Table 1: Clinical and biochemical characteristics of all patients } \\
Characteristics & Group I $(n=144)$ & Group II $(n=70)$ & $p$-value \\
\hline Age (year) & $39.9 \pm 10.39$ & $34.5 \pm 13$ & 0.0029 \\
BMI (kg/m $\left.{ }^{2}\right)$ & $26.44 \pm 4.75$ & $23.51 \pm 4.43$ & 0.0000 \\
FBS (mg/dl) & $106.08 \pm 38.75$ & $97.06 \pm 31.77$ & 0.072 \\
T. cholesterol (mg/dl) & $198.18 \pm 43.25$ & $182.23 \pm 46.87$ & 0.0181 \\
LDL-c (mg/dl) & $124.95 \pm 34.91$ & $115.13 \pm 39.6$ & 0.0803 \\
HDL-c (mg/dl) & $40.5 \pm 10.62$ & $41.16 \pm 11.56$ & 0.690 \\
TGs (mg/dl) & $166.96 \pm 119.49$ & $132.14 \pm 81.79$ & 0.0134 \\
AST (IU/l) & $34.71 \pm 30.53$ & $21.59 \pm 4.97$ & 0.0000 \\
ALT (IU/l) & $52.93 \pm 35.17$ & $22.3 \pm 5.97$ & 0.0000 \\
Serum bilirubin (mmol/l) & $0.93 \pm 0.32$ & $0.89 \pm 0.35$ & 0.710 \\
Serum albumin (gm/dl) & $3.8 \pm 0.99$ & $3.9 \pm 0.98$ & 0.540 \\
Prothrombin time (sec) & $12.9 \pm 0.81$ & $12.7 \pm 0.72$ & 0.74 \\
\hline
\end{tabular}

BMI: Body mass index; FBS: Fasting blood sugar; T. cholesterol: Total cholesterol; LDL-c: Low density lipoprotein cholesterol; HDL-c: High density lipoprotein cholesterol; TGs: Triglycerides, AST: Aspartate aminotransferase; ALT: Alanin aminotransferase

Table 2: Clinical and biochemical characteristics of groups la and lb patients with normal and high BMI

\begin{tabular}{lccc} 
Characteristics & \multicolumn{2}{c}{ Group I } & $p$-value \\
\cline { 2 - 4 } & la normal BMI $(n=55)$ & lb high BMI $(n=89)$ & 0.1869 \\
\hline Age (year) & $38.42 \pm 10.59$ & $40.8 \pm 10.22$ & 0.00000 \\
BMI (kg/m $\left.{ }^{2}\right)$ & $22.28 \pm 2.34$ & $29.01 \pm 3.97$ & 0.00967 \\
FBS (mg/dl) & $96.24 \pm 30.5$ & $112.16 \pm 42.1$ & 0.1862 \\
T. cholesterol (mg/dl) & $191.74 \pm 49.53$ & $202.17 \pm 38.62$ & 0.37698 \\
LDL-c (mg/dl) & $121.62 \pm 35.46$ & $127.02 \pm 34.6$ & 0.14 \\
HDL-c (mg/dl) & $42.2 \pm 11.45$ & $39.44 \pm 9.99$ & 0.1599 \\
TGs $(\mathrm{mg} / \mathrm{dl})$ & $148.51 \pm 129.14$ & $178.37 \pm 112.35$ & 0.9842 \\
AST (IU/l) & $34.76 \pm 16.99$ & $34.67 \pm 36.57$ & 0.9749 \\
ALT (IU/l) & $53.04 \pm 28.27$ & $52.86 \pm 38.98$ & 0.301 \\
Serum bilirubin (mmol/l) & $0.904 \pm 0.22$ & $0.898 \pm 0.21$ & 0.46 \\
Serum albumin $(\mathrm{gm} / \mathrm{dl})$ & $3.8 \pm 0.71$ & $3.79 \pm 0.92$ & 0.5 \\
Prothrombin time $(\mathrm{sec})$ & $12.9 \pm 0.81$ & $12.7 \pm 0.97$ & \\
\hline
\end{tabular}

\begin{tabular}{lccc} 
& Table 3: Liver ultrasound results in group I patients \\
Liver ultrasound & Group I $(n=144)$ & Group la $(n=55)$ & Group Ib $(n=89)$ \\
\hline Normal & $55(38 \%)$ & $33(60 \%)$ & $22(25 \%)$ \\
Bright liver & $43(30 \%)$ & $10(18 \%)$ & $33(37 \%)$ \\
Periportal fibrosis & $30(21 \%)$ & $3(5.5 \%)$ & $27(30.5 \%)$ \\
Fatty hepatomegaly & $12(8 \%)$ & $8(14.5 \%)$ & $4(4.5 \%)$ \\
Liver cirrhosis & $4(3 \%)$ & $1(2 \%)$ & $3(3 \%)$ \\
\hline
\end{tabular}

The data of this study have been shown in Tables 1 to 3 and will be discussed in discussion section in relevant conditions.

\section{DISCUSSION}

Khat is one of the most common forms of drug use and abuse in many East Africa nations (Kenya, Ethiopia) and Arabian Peninsula (Yemen). It involves chewing parts of the khat plant. Khat use has increased steadily over the last 50 years and has become a problem of significant social and medical importance. Because of it's social acceptability and euphoriant effects, khat chewing often plays a dominant role in celebrations, meetings, marriages and other gatherings.

Hepatic cirrhosis of unknown etiology has been noted in khat users; poor diet and the potential hepatotoxic effects of khat Tannians may be contributing factors. ${ }^{13}$ The evidence, however, is often based on limited numbers of case reports and animal studies.

The present study showed that khat chewing is very common in Yemen, and liver toxicity in the form of either elevated liver enzymes and/or abnormal liver finding during abdominal ultrasound was highly prevalence in Yemeni people $(67 \%)$ of the studied population sample, and up to liver cirrhosis in 3\% of cases. Sixty-nine percent of a total 178 males, and $58 \%$ of a total 36 females, showed hepatic insult with almost similar sex incidence.

The current study showed that patients with liver damages (either by elevated liver enzymes or liver ultrasound scan or both) were significantly older in age than 
participants without hepatic involvement $(p=0.00295)$. This may explain in part why 70 khat users had no evidence of hepatic injury. This is because the amount of khat chewed per user is about 100 to 200 gm of leaves and stems over 3 to 4 hours. The tender leaves and stems, which lose their potency/day after harvest, are chewed and the juice is swallowed, large amounts of liquids are consumed while chewing because of dryness induced by the plant. ${ }^{18}$

There are three main alkaloids present in khat leaves; S-(-)-cathinone (S- $\alpha$-aminopropriophenone), norpseudoephedrine (cathine) and norephedrine. ${ }^{9}$ There are also small amount of ethereal oil, sterols and triterpenes, together with $5 \%$ protein which has insignificant nutritional value. Ascorbic acid is also present in the leaves. ${ }^{19}$

Apart from tannin, these substances are unlikely to contribute to the biological effect of khat. ${ }^{9}$ S-(-)-cathinone is relatively unstable and decomposes within a few days of picking or if the leaf is dried into (+)-norpseudoephedrine and norephedrine. Thus, only freshly picked leaves have the full efficacy.

Short- and long-term toxicity of khat leaves have been tested in laboratory animals for a period of 6 months. The liver biochemical and histological parameters were found to be significantly altered with a profound impact on the alkaline phosphatase and alanine aminotransferase (ALT) plasma levels throughout the treatment period. ${ }^{16,17}$

Results of the current study showed that group I patients with liver damages (144 patients), 127 (88\%) of them had elevated liver enzymes mainly ALT (ALT - 52.93 \pm 35.17 , and AST $-34.71 \pm 30.53 \mathrm{IU} / \mathrm{l}$ ) and only 17 patients (12\%) had normal levels of liver enzymes (in the presence of abnormal liver ultrasound finding).

In laboratory, animals tested after 6 months period treatment with khat, histopathological analysis of the liver showed evidence of congestion of the central veins as well as acute hepatocellular degeneration and regenerative activities, with portoportal fibrosis, a vasoconstrictor action of cathinone would also contribute to this liver pathology. ${ }^{16,17}$

Liver ultrasound results of our group I patients showed that 43 patients (30\%) showed bright liver, 30 patients (21\%) showed periportal fibrosis, 12 patients (8\%) showed hepatomegaly picture of fatty infiltration and four patients (3\%) had liver cirrhosis. Fifty-five patients (38\%) with elevated liver enzymes showed normal liver in ultrasound.

In confirmation of the hepatotoxicity of khat ingestion, a case report describe an East African man with a regular khat chewing habit who developed jaundice as a result of acute hepatitis. ${ }^{14}$ Another study described jaundice and deranged liver function based on biopsy histology and serum biochemistry in seven UK men of Somali origin who were regular khat chewers, and all were denied any alcohol consumption and no other etiological factors could be identified. ${ }^{15}$ The results of the current study are in agreement with the previous case reports, because of all of our patients tested negative for HCV and HBV. And, khat chewing was the only positive factor related to liver injury.

The results of the current study showed that 34 patients (30\%) had bright liver and 30 patients (21\%) had hepatomegaly among khat users patients with hepatic damages (group I patients). And, group I patients showed BMI significantly higher than group II patients without liver involvement (BMI $26.44 \pm 4.75$ vs $23.51 \pm 4.43 \mathrm{~kg} / \mathrm{m}^{2}$ in groups I and II respectively).

The presence of bright liver and fatty hepatomegaly is the most common etiology of nonalcoholic fatty liver disease (NAFLD) because other possible causes of liver damages were excluded. We further subdivide group I patients into two subgroups according to BMI to exclude effect of obesity in our results, and to clarify if other factor than khat chewing had a role in the finding of the present study.

Group I patients with hepatic damages were subdivided into group Ia (55 patients) with normal BMI and group Ib (89 patients) with variable degree of obesity.

There were no significant difference between liver enzymes (ALT and AST) between these subgroups ( $p=0.97495$ and 0.98417$)$.

Our results have mostly supported the notion that khat is mainly responsible factor for the changes in lipid profile. And, this in agreement with the documented severe adverse vascular effect of khat as, myocardial infarction, hypertension and cerebrovascular ischemia. ${ }^{12}$

Liver ultrasound showed abnormal finding in 22 (40\%) patients with normal BMI, and this finding support our exclusion of the obesity to be the responsible factor for hepatic injury in those khat user patients. The influence of obesity on the liver ultrasound results can not be ignored but the presence of fatty infiltration in liver ultrasound (in the form of bright liver and fatty hepatomegaly) in 32.5\% of patients with normal BMI compared to $41.5 \%$ of patients with variable degree of obesity might put the khat in the main spot. Also, the presence of periportal fibrosis in 5.5\% of patients and liver cirrhosis in $2 \%$ of patients with normal BMI support this conception.

In 2010, Chapman et al reported an observation on six patients who use khat regularly, presented over a 5-year period with severe acute hepatitis, all had similar histopathological findings characterized by multilobular necrosis; two of them had a background of chronic liver disease. Five out of the six patients underwent orthotropic liver transplantation. A number of factors point to khat 
ingestion as the cause of liver injury in these patients. Chapman et al found no other cause except khat taking for their liver disease. A high concentration of cathinone was identified in a sample of damaged liver tissue from our patients, khat-related hepatotoxicity has been demonstrated in animals, and the histopathologic changes in liver mirror those induced in humans by ingestion of the drug Ecstasy, another amphetamine-like compound. ${ }^{13}$

The result of the present study showed that the khat users patients with positive finding of hepatic damages are comparatively older. Even the four patients who found to have liver cirrhosis were older than other patients, age was $54.75 \pm 7.12$ years in liver cirrhosis patients, compared to $39.9 \pm 10.39$ years in other patients with positive hepatic injury, however, the age of patients without hepatic insult was $34.5 \pm 13$ years.

This suggests that a cumulative effect of khat may induce liver damages.

It has been reported that the mechanism of khat-related hepatotoxicity is unknown, there were no compelling features of an immunoallergic or autoimmune process, although both should be considered, and a high concentration of cathinone was detected in the liver of a patient 3 weeks after the patient's last use of khat, suggesting that drug accumulation may be important. However, it has proposed that liver injury is unrelated to contaminants, such as herbicides, pesticides, heavy metals or toxigenic fungi. ${ }^{13}$

The present study showed, hepatotoxic effect of khat chewing in Yemeni people. But, this study differ from what has been reported by Schiff et al who said that, when doctors see liver problems associated with a drug as widely used as khat, 'it usually means that the particular person had metabolized it differently, because otherwise you should see it in thousands of people'. ${ }^{20}$ But, the present study that included a considerable numbers of sample size showed that $67 \%$ of khat users had variable degrees of hepatic injury.

The current study answered some of questions raised by Al-Motarreb et al who commented that 'a confounding issues, other than khat chewing need to be eliminated as causative factors in many diseases of peripheral organs in khat users, such as concomitant tobacco smoking, alcohol consumption, coexisting disease states, diet and obesity'. ${ }^{12}$

We should point out some limitations of the present study. The first, liver biopsy was not taken to confirm ultrasound results in all patients. The next, we did not report dose and exact duration of khat intake because it was a retrospective study.

The chewing of khat is a well-established social habit in the Yemen and other parts of the Middle East and East
Africa. While the psychological effects of chronic khat use have been the subject of much debate on its influence of social structure, there is now mounting evidence of concern over the health effects on a wide range of peripheral organs. Hepatotoxic effect of khat is common and even be related to development of liver cirrhosis. Confounding issues other than khat chewing were eliminated in the present study as causative factors, such as alcohol consumption and coexisting disease. Also, the effect of obesity was evaluated.

\section{REFERENCES}

1. Manghi RA, Broers B, Khan R, Benguettat D, Khazzaal Y, Zulino DF. Khat use: Lifestyle or addiction? J Psychoactive Drugs 2009;41:1-10.

2. Kalix P. Catha edulis, a plant that has amphetamine effects. Pharm World Sci 1996;18:69-73.

3. Laswar AK, Darwish AK. Prevalence of cigarette smoking and khat chewing among Aden University medical students and their relationship to BP and body mass index. Saudi J Kidney Dis Transpl 2009;20:862-66.

4. Ageely HM. Prevalnce of khat chewing in college and secondary (high) school students of Jaza region. Saudi Arabia. Harm Reduct J 2009;6:11.

5. Tesfaye R, Byass P, Wall S, Berhane Y, Bonita R. Association of smoking and khat (Catha edulis forsk) use with high blood pressure among adults in Addis Ababa, Ethiopia 2006. Preventing Chronic Disease 2008;5:A89.

6. kussim S, Croucher R. Khat chewing amongst UK resident male Yemeni adults: An exploratory study. Inte Den J 2006;56: 97-101.

7. Benture Y, Bloom-Krasik A, Raikhlin-Eisenkraft B. Illicit cathinone (Hagigat) poisoning. Clin Toxicol (Phila) 2008; 46:206-10.

8. Holligan A. Khat use spreads to British Youth. BBC World service, online news March 16, 2009. Available from: http:// news.bbc.co.uk/1/hi/7942432.stm.

9. Kalix P. Cathinone, a natural amphetamine. Pharmacol Toxicol 1992;70:77-86.

10. Deyessa N, Berhane Y, Alem A, Hogberg U, Kullgren G. Depression among women in rural Ethiopia as related to socioeconomic factors: A community-based study on women in reproductive age groups. Scand J Public Health 2008;36:589-97.

11. WHO expert committee on drug dependence. Thirty fourth report. WHO Technical Report Series, Report 942, Geneva 2006.

12. Al-Motarreb A, Al-Habori M, Broadley KJ. Khat chewing, cardiovascular disease and other internal medicine problems: the current situation and direction for future research. J Enopharmacol 2010;132:540-48.

13. Chapman MH, Kajihara M, Borges G, O’Beirne J, Patch D, Dhillon AP, et al. Severe, acute liver injury and khat leaves. New Engl J Med 2010;362:1642-43.

14. Brostoff M, Ptymen C, Birns J. Khat-A noval cause of drug induced hepatitis. Eur J Intern Med 2006;17:383.

15. Peevers CG, Moorghen M, Collins PL, Gordon FH, McCune CA. Liver disease and cirrhosis because of khat chewing in UK Somali men: A case series. Liver Int 2010 Sep:30(8):1242-43. 
16. Al-Habori M, Al-Aghbari A, Al-Mamary M, Baker M. Toxicological evaluation of Catha edulis leaves: A long term feeding experiment in animals. J Ethnopharmacol 2002;83: 209-17.

17. Al-Mamary M, Al-Habori M, Al-Aghbari A, Baker M. Investigation into the toxicological effects of Catha edulis leaves: A short term study in animals. Phytother Res 2006;16:127-32.

18. Randall T. Khat abuse fuels Somali conflict, drains economy. JAMA 1992;269:12-15.

19. Raman R. Catha edulis forsk, geographical dispersal, botanical, ecological and economical aspects with special references to Yemen Arab Republic. PhD thesis, University of Gottingen. Germany 1983.

20. Schiff E. Chewing khat linked to severe liver damage. Herb appears to lead to liver failure in some British researchers say. Health day report, online news April, 29.2010.

\section{ABOUT THE AUTHORS}

\section{Mona Abd Elmonem Hegazy}

Faculty of Medicine, Department of Internal Medicine, Cairo University, Cairo, Egypt

Correspondence Address: 166, 26th July Street, Spinx Square, Elmohandsin Giza, Egypt, e-mail: monahegazy@hotmail.com

\section{Nehad Mohamed Tawfik}

Faculty of Medicine, Department of Internal Medicine, Cairo University, Cairo, Egypt

\section{Hoda Abd-Elstar Elrawi}

Faculty of Medicine, Department of Internal Medicine, Cairo University, Cairo, Egypt 